\title{
Comments to the Article "The Behavior of Radial Electric Field and Toroidal Rotation in the Edge Plasma of Divertor Tokamak" by A. H. Bekheit Published in J. Fusion Energy (2010) 29, p. 443-446
}

\author{
Alexander Romannikov
}

Published online: 29 January 2011

(C) Springer Science+Business Media, LLC 2011

I would like to pay attention that the main idea and conclusions of the article "The Behavior of Radial Electric Field and Toroidal Rotation in the Edge Plasma of Divertor Tokamak" by A. H. Bekheit published in J. Fusion Energy (2010) 29, p. 443-446 are practically identical to the article "Certain considerations concerning the nature of radial electric field and toroidal rotation velocity profile in tokamak plasma" by A. Romannikov, published in Plasma Phys. Control. Fusion, 49 (2007) 641-647, without any necessary reference.

These coincidences, for example, are:

1. In the text of Romannikov's article on the bottom of p. 642 and in the text of Bekheit's article after Eq. (3); 2. $\mathrm{E}_{\mathrm{r}}^{\mathrm{add}}\left(\mathrm{r}^{*}\right)$ in the Eq. (5) of Romannikov's article, p. 643 $\left(\mathrm{E}_{\mathrm{r}}^{\mathrm{add}}\left(\mathrm{r}^{*}\right)\right.$ is consequence mainly of "The $\boldsymbol{E}$ and $\boldsymbol{B}$ components of the electromagnetic field in the instantaneous inertial coroating frame in which the material medium is at rest can be easily related [15] to the $\boldsymbol{E}$ and $\boldsymbol{B}$ components in the laboratory frame. In our case, it is possible to consider the corotating frame also as the instantaneous inertial frame [16, 17].”, p. 642) is practically the $E_{r}^{(1)}$ in the Eq.(5) of Bekhe's article, P. $444\left(E_{r}^{(1)}\right.$ is consequence mainly of "The radial electric field "Er"' and magnetic field ' $\mathrm{B}$ "' components of the electromagnetic field in the instantaneous inertial corotating frame in which the material medium is at rest can be easily related to the radial electric field " $E r$ " and magnetic field " $\mathrm{B}$ " components in the laboratory frame [10]. In our case, it is possible to consider the corotating frame also as instantaneous inertial frame [11, 12]", p. 444);

3. Items 4,5 and 7 in Conclusion of Romannikov's article, p. 646 and items 2, 3 and 5 in Conclusion of Bekheit's article, p. 445;

4. References [14, 15, 16, 17 and 20] of Romannikov's article, p. 647 and references [9, 10, 11, 12 and 14] of Bekheit's article, p. 446; etc.

Especially, I would like to pay attention to references $[16,17]$ of Romannikov's article, p. 647 and references [11, 12] of Bekheit's article, p. 446. References to the French mathematician Mo T. C. 1970 J. Math. Phys. 11, 2589 and to Van Bladel J., 1984, "Relativity and Engineering" (Heidelberg: Springer) are in tokamak physics publications only in three articles: A. N. Romannikov, Plasma Phys. Control. Fusion, 49 (2007) 641-647; A. N. Romannikov, JETP (2009), Vol. 108, No. 2, pp. 340-348; and A. H. Bekheit J. Fusion Energy (2010) 29, pp. 443-446.

A. Romannikov ( $\square)$

ITER Agency, Russian Research Center «Kurchatov Institute», pl. Kurchatova I., 1, Moscow 123182, Russia

e-mail: a.romannikov@iterrf.ru 